\title{
Breast Cancer Outcomes Among Older Women
}

\author{
HMO, Fee-for-service, and Delivery System Comparisons \\ Anna Lee-Feldstein, PhD, Paul J. Feldstein, PhD, Thomas Buchmueller, PhD, Gale Katterhagen, MD
}

\begin{abstract}
OBJECTIVE: To analyze the relationship of health insurance status and delivery systems to breast cancer outcomes - stage at diagnosis, treatment selected, survival - focusing on comparisons among women aged 65 or more having Medicare alone, Medicare/Medicaid, or Medicare with group model HMO, non-group model HMO, or private fee-for-service (FFS) supplement.
\end{abstract}

DESIGN: Retrospectively defined cohort from Sacramento, Calif, regional cancer registry.

SETTING: Thirteen-county region in northern California with mature managed care market.

PATIENTS: Female invasive breast cancer patients aged 65 or more $(N=1,146)$, diagnosed 1987-1993.

MEASUREMENTS AND MAIN RESULTS: Health insurance was determined from hospital records. Outcomes were analyzed with multivariate regression models, controlling for age, ethnicity, time, and SES measures. Stage I diagnosis was more likely among group model HMO patients than among private FFS insured (odds ratio [OR], 1.42; 95\% confidence interval [CI], 0.84 to 2.40). Stage I tumors were significantly less likely for Medicaid patients (OR, 0.50; 95\% CI, 0.31 to 0.82). Use of breast-conserving surgery plus radiation (BCS+) varied significantly by hospital type (including HMO-owned and various-sized community hospitals) and time. Survival of patients with private FFS, group-, and non-group model HMO insurance was not significantly different, but was for those with Medicaid or Medicare alone.

CONCLUSIONS: This study sheds new light on the relationship of insurance to stage and survival among older breast cancer patients, highlighting the importance of distinguishing types of HMOs and types of FFS plans. These outcomes do not differ significantly between women with Medicare who are in HMOs and those with private FFS supplemental insurance. However, patients with Medicare/Medicaid or Medicare alone are at risk for poorer outcomes.

Received from the Center for Health Policy and Research, Department of Medicine, College of Medicine (ALF); Graduate School of Management, University of California, Irvine, Calif (PJF, TB); and Office of Medical Director, Cancer Center, Tacoma General Hospital, Tacoma, Wash (GK).

Address correspondence and reprint requests to Dr. LeeFeldstein: Health Policy and Research, 100 Theory, Suite 110, University of California Irvine, Irvine, CA 92697-5800.
KEY WORDS: health insurance; hospital type; breast cancer; stage; treatment; survival. J GEN INTERN MED 2001;16:189-199.

$\mathrm{R}$ esearch into the effectiveness of HMOs among Medicare patients aged 65 or more is important since the number of Medicare beneficiaries enrolled in HMOs has grown from 3.6 million in 1995 to 5.6 million in 1997, ${ }^{1,2}$ and leading Medicare reform proposals would expand the use of managed care. Although managed care potentially can improve health care quality by facilitating prevention, screening and treatment interventions, recently there has been growing concern about quality of care provided by HMOs.

Breast cancer is a significant disease, for which data are available to research the performance of HMOs among Medicare patients. Among U.S. women aged 65 or more, breast cancer is the most common site of new invasive cancers and the second most common cause of cancer mortality, with an estimated 87,500 new cases and 26,000 deaths in $1996 .^{3,4}$ Further, early detection and treatment of breast is associated with improved survival. In California (current study site) breast cancer mortality among older women fell about 9\% from 1988 to $1996,{ }^{5}$ owing in part to increased use of screening mammography and earlier stage at diagnosis. ${ }^{6-8}$

Major clinical trials in the 1980s demonstrated that survival was similar for early stage breast cancer patients treated with breast-conserving surgery (BCS) or mastectomy. ${ }^{9-11}$ Subsequently the 1990 NIH Consensus Conference $^{12}$ recommended BCS plus radiation therapy as preferable treatment for the majority of Stage I or II breast cancers without reference to age of the patient. The proportion of early stage breast cancers treated with BCS among older California women increased from $28 \%$ in 1988 to $43 \%$ in $1992 .^{7}$

The present multivariate study of breast cancer patients aged 65 and older residing in northern California investigates quality of breast cancer care by examining the relationship of six types of health insurance to three outcomes: 1) stage at diagnosis; 2) treatment modality selected; and 3) survival. The study also considers breast cancer outcomes relative to hospital type while adjusting for other factors known to be associated with these outcomes (age, ethnicity, education, neighborhood class, and time period). ${ }^{13-23}$ 
This paper explores the following hypotheses: 1) Among Medicare beneficiaries with breast cancer, the odds of early stage diagnosis differ between those with private insurance (private FFS, group-model HMO, non-group-model HMO) and those without, and within the private insurance group stage at diagnosis varies by insurance type; 2) Among Medicare patients diagnosed at an early stage, use of BCS plus radiation varies over time and with hospital type; and 3) Among Medicare beneficiaries having early stage breast cancer, there are insurance-related differences in survival, with better outcomes associated with private insurance.

In testing these hypotheses the current study extends and improves upon existing literature in several ways. First, few prior studies of breast cancer stage at diagnosis among Medicare beneficiaries compared HMO and FFS patients, and none employed the detailed categorization of insurance coverage found here. ${ }^{15,18,23,24}$ In an earlier study, Riley et al. ${ }^{18}$ used a heterogeneous FFS group, including Medicare patients with private FFS supplemental insurance as well as those having only Medicare and those with Medicaid; but this FFS grouping biases inferences related to detection and treatment in favor of HMOs. In addition, the current study distinguishes between group and non-group model HMOs, which is important since they differ in physician culture, organization, and financial incentives. While much early research involving HMO treatment outcomes among older females is based on data from group model plans, growth in HMO enrollment in the 1980s and 1990s was almost entirely due to non-group model plans. ${ }^{25}$

Two previous investigators ${ }^{22,23}$ considered the relationship between insurance coverage and breast cancer treatment among Medicare patients (again, using the catch-all FFS category), lacking controls for hospital type. Conversely, many others considered the relationship between hospital characteristics and breast cancer treatment for women aged 65 or more, without accounting for differences in insurance coverage among patients. ${ }^{17,19-}$ 21,26-29 The present study improves upon most of this literature by including both insurance and hospital type, using a hospital typology which includes a category for HMO-owned hospitals.

A final advantage of this study relative to earlier research is the time period (1987-1993) to which the current data pertain, providing new HMO/private FFS comparisons regarding diagnosis and survival among older women. The timing of the present study is also well-suited for studying breast cancer outcomes, as diagnosis of study subjects encompasses both the NIH Consensus Conference year and inception of Medicare coverage for biennial mammograms (1990).

\section{SUBJECTS AND METHODS}

\section{Study Subjects}

Patients for this study were identified through the Cancer Surveillance Program, Region 3 (Sacramento,
Calif), one of ten population-based regional registries in the statewide California Cancer Registry. All subjects were residents of the thirteen county region including Alpine, Amador, Calaveras, El Dorado, Nevada, Placer, Sacramento, San Joaquin, Sierra, Solano, Sutter, Yolo, and Yuba counties, a mixture of urban and rural areas in northern California with an estimated total population of 2.6 million in 1990. The Region 3 registry includes case reports for all resident cancer patients seen in hospitals and clinics in the 13-county region. In addition, case reports from other regions and from physicians' offices and other facilities within the region ensure virtually $100 \%$ ascertainment of incident cancer cases among regional residents. ${ }^{30}$

Using data from a single geographic area has both pros and cons, although the former significantly outweigh the latter. On the positive side, it reduces the possibility that geographic differences in practice and treatment patterns related to differences in physician training and other factors will confound the relationship between insurance status and cancer-related outcomes. This is particularly important for the current study since there is tremendous variation in HMO penetration across the country. ${ }^{2}$ By focusing on northern California, this study examines a mature managed care market that is indicative of the direction in which one might expect other managed care markets to evolve. The obvious limitation of this approach is that, to the extent that northern California is unique relative to the rest of the country in HMO practices and policies, the results may not generalize to all other settings.

Study subjects were women aged 65 or greater diagnosed 1987-1993 with invasive breast cancer (ICDO diagnostic codes C50.0 through C50.9), ${ }^{31}$ having no known prior cancer at any site and receiving all or part of their first course of treatment at the reporting hospital. Because of the cost of obtaining insurance data, the analysis was conducted on a sample of available patients. All nonHispanic African American (African American), Hispanic, and non-Hispanic Asian/other (Asian) patients were included to ensure sufficient cell sizes for estimating ethnic differences in stage, treatment, and mortality. In addition, a $28 \%$ random sample of non-Hispanic Whites (Whites) was drawn. Excluded from the study were 2 patients diagnosed in hospitals outside northern California, 34 reported by non-hospital sources, 20 not treated at the reporting hospital, and 137 whose tumor could not be staged due to incomplete information. The total sample size was 1,146 ; for some analyses the sample was smaller (explained below).

The first study outcome, stage at diagnosis, was determined from information on tumor size, nodal involvement, and metastases, following guidelines from the American Joint Commission on Cancer. ${ }^{32,33}$ Stage I tumors are confined to the breast and $\leq 2 \mathrm{~cm}$ in diameter; Stage II tumors either are $>2 \mathrm{~cm}$ or involve moveable ipsilateral axillary nodes or both. Stage III and IV tumors are more 
extensive, involving fixed axillary nodes, direct extension to the chest wall or skin, or distant metastases.

Treatment outcomes considered were: 1) breastconserving surgery (BCS) without radiation; 2) breastconserving surgery with radiation (BCS+); 3) mastectomy; or 4) other/ unknown. BCS surgeries include nipple resection, lumpectomy, excisional biopsy, wedge resection, quadrantectomy, segmental mastectomy, tylectomy, and partial mastectomy. The mastectomy category included total (or simple), modified radical, and radical mastectomy.

For survival analyses, follow-up information (vital status, date of death or last contact) through December 1996 was obtained from hospital tumor registrar reports, and through linkage with annual California mortality files. For those not known to have died, date of last contact was also updated by linking with files from the California Department of Motor Vehicles. The mean follow-up time from diagnosis date was 51.2 months. At 30,60 , and 90 months after diagnosis, respectively, 79\%, $39 \%$, and $11 \%$ of Stage I and II patients were at risk of dying.

The cancer registry provided standard data items for this study, including age at diagnosis, ethnicity, tumor data, type of hospital, and treatment variables. No data were available from this source regarding the main independent variable of interest in this study: health insurance status at diagnosis. This information was determined from hospital records for 98\% of patients. Study subjects were assumed to have their current insurance in effect during the year preceding diagnosis, when screening might detect the disease. The likelihood of switching health plans after diagnosis is estimated to be minimal (Strombom B, Buchmueller T, Feldstein P. "Switching costs, price sensitivity, and health plans," unpublished, May 2000). Insurance status was coded as follows: 1) Medicare alone; 2) Medicare/private FFS; 3) Medicare/Medicaid; 4) Medicare/group model HMO; 5) Medicare/non-group model HMO; and 6) other (including unknown). The group HMO category consists of one plan, which is one of the largest both in the region and nationally. A total of 9 different health plans are represented in the non-group HMO category, and the largest of these accounts for $74 \%$ of the observations in this category. Due to consolidation in the industry, the number of distinct HMOs in the dataset declines over time - by 1993 five non-group HMOs were represented. It is important to determine whether HMOs place greater emphasis on early diagnosis than FFS plans and whether certain types of HMOs (profit/non-profit and group/nongroup models) are more effective in this regard. Thus, the ability to differentiate between so many types of insurance coverage is a key factor which distinguishes this analysis from previous studies.

Although the cancer registry has no information on patient socioeconomic status (SES), which may affect on various outcomes in this study. ${ }^{6,34-41}$ there is information on each patient's census block-group at the time of diagnosis. Thus, two block-group variables from the U.S. 1990 census were included as controls for SES: percent blue collar, and percent age 25 or over having a college degree. ${ }^{35,42}$ Based on the percentage of block-group workers employed in working class occupations, a patient's neighborhood class was designated as blue collar $(\geq 66 \%)$ or non-blue collar. ${ }^{42}$ The observed range of educational levels was divided into tertiles, yielding a designation of least, moderately, or most educated for each patient.

To study the relationship between hospital type and treatment selected, hospitals providing data for this study were classified as follows: large community ( $n=$ $13,270+$ licensed beds), medium community ( $n=10$, 170 to 269 beds), moderately small community ( $n=17$, 95 to 169 beds), very small community ( $n=8, \leq 95$ beds), rural $(n=7)$, teaching $(n=8)$, and HMO-owned hospitals $(n=10) .^{43,44}$

\section{Analysis}

Statistics for this study were computed with SAS procedures. ${ }^{45,46}$ The $\chi^{2}$ test was used to evaluate differences in the distribution of categorical variables (ethnicity, education, neighborhood class, stage at diagnosis) by type of health insurance; the Kruskal-Wallis analysis of variance by ranks was used to test for differences in age by insurance type. In the model-building process important measurable risk factors were first identified, and the best fitting multivariate regression model containing these variables was determined. Two-factor interactions significant at the .05 level were included. Logistic regression models were tested for goodness-of-fit; in each case there was good agreement between observed values and those predicted from the models. ${ }^{47}$

The analysis began with an examination of the relationship between patient insurance status and stage at diagnosis of breast cancer, controlling for differences in age, ethnicity, and SES measures. The basic model is given by:

(1) Stage = F (insurance, age, ethnicity, education, neighborhood class).

The outcome variable, or stage at diagnosis, was estimated by a polytomous response logistic regression model. ${ }^{47,48}$ The model compared patients with Stage I, Stage II tumors $\leq 2.5 \mathrm{~cm}$, and Stages III-IV, respectively, against the baseline group (patients with Stage II tumors $>2.5 \mathrm{~cm}$ ). This is similar to a series of dichotomous response logistic regressions, each one estimating the probability of diagnosis at a specific stage versus diagnosis at the baseline (e.g., Stage I vs Stage II with tumor $>2.5 \mathrm{~cm}$ ). For a specific outcome (stage), an odds ratio greater than 1.0 for a particular level of independent variable denotes increased likelihood of diagnosis at that stage compared to the reference level. 
The second outcome examined was treatment selected, which was measured as a dichotomous variable - BCS+ vs mastectomy. The analysis was limited to patients diagnosed at Stage I or II, as $\mathrm{BCS}+$ is recommended treatment for early stage breast cancer. ${ }^{12}$ The independent variables of primary interest are insurance coverage and hospital type. The hospital effects were allowed to vary over time to account for possible differences in the diffusion of recommended practices. The treatment model is given by:

(2) Treatment = F (insurance, hospital type, stage, control variables, time $\mathrm{x}$ hospital type),

where the control variables are the same as in the stage at diagnosis model. Because HMO-owned hospitals treated solely patients with group model HMO insurance and hospital type was the significant factor in this analysis, insurance type was set equal to zero for these patients.

Thirdly, the analysis centered on the relationship of survival (i.e., all cause mortality and breast cancer mortality) to insurance type, hospital type, stage at diagnosis, and treatment, controlling for ethnicity, age and SES variables. A simple representation of the model is given by:

(3) Survival = F (insurance, hospital type, stage, treatment, control variables).

Survival was estimated by Cox proportional hazards multivariate regression models for censored survival data. ${ }^{49,50}$ Since patients seen at HMO-owned hospitals had only group model HMO insurance and insurance type was the significant factor in this analysis, hospital type was set equal to zero for these patients. In estimating

Table 1. Unadjusted Summary Statistics for 1,146 Breast Cancer Patients Age 65 or Greater at Diagnosis, by Type of Health Insurance

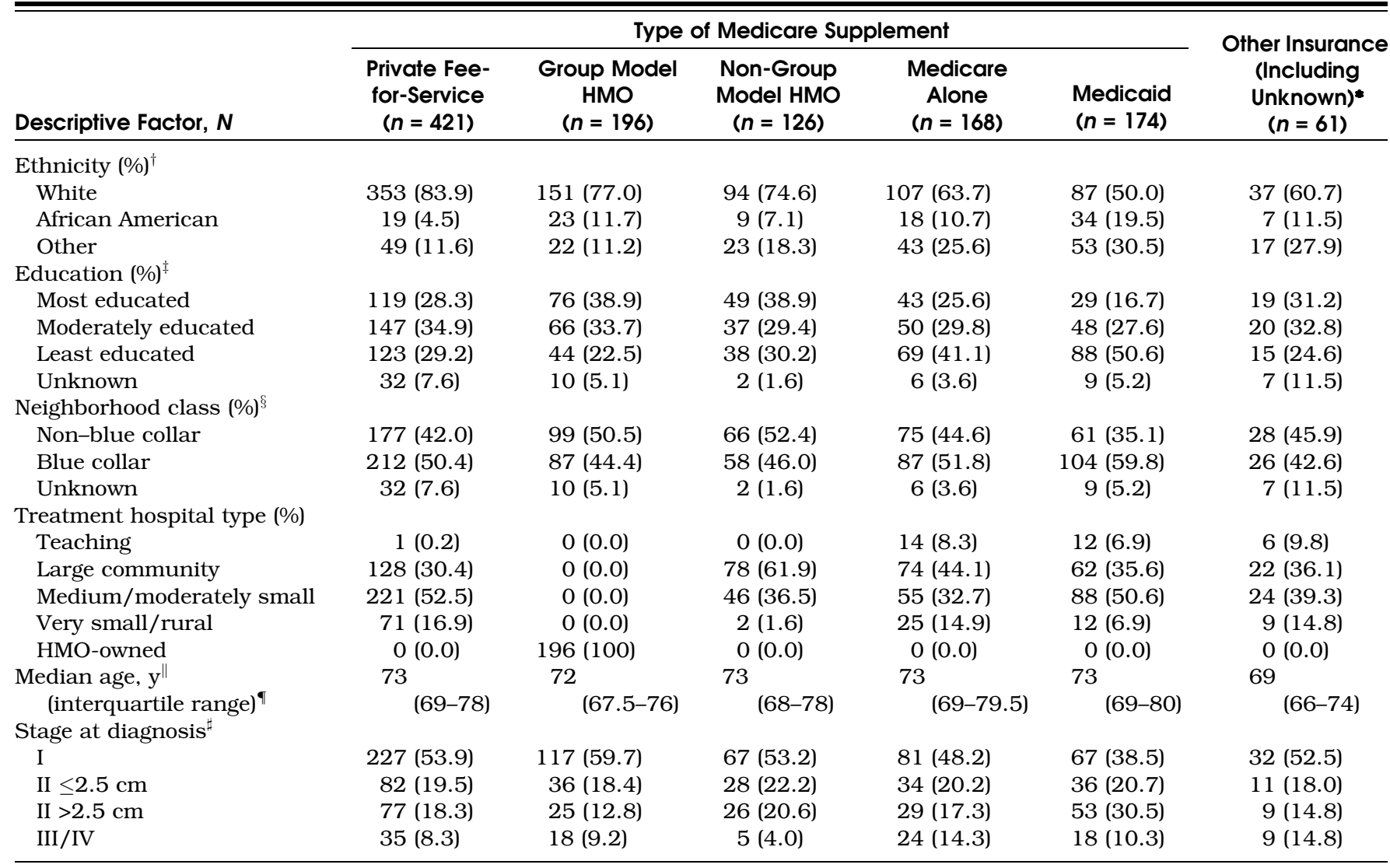

* This category includes 61 patients not on Medicare (private, public assistance, or no insurance).

${ }^{\dagger}$ Ethnicity differs significantly by type of insurance $(\mathrm{P}<.001)$. The "other" category includes Hispanic and Asian.

${ }^{\ddagger}$ Education (based on average educational level of patient’s 1990 census block group) differs significantly by type of insurance (P <.001).

${ }^{\S}$ Neighborhood class (based on \% blue collar workers in patient's 1990 census block group) differs significantly by type of insurance (P = .006). The line for Unknown Neighborhood Class is identical to the line for Unknown Education. This is due to the fact that census tract was unknown for 32 individuals out of the 1,146, and neither Neighborhood Class nor Education could be estimated for these individuals.

"Median age differs significantly by type of insurance (Kruskal-Wallis analysis of variance by ranks, $\mathrm{P}<.001$ ).

"Interquartile range is interval from $25^{\text {th }}$ to $75^{\text {th }}$ percentile (middle $50 \%$ of observations).

\#Stage at diagnosis differs significantly by type of insurance $(\mathrm{P}=.001)$. 
breast cancer mortality, those dying of other causes were censored at date of death. In addition, Kaplan-Meier estimates of survival ${ }^{51}$ by time since diagnosis were calculated; the log-rank test ${ }^{52}$ was used to assess differences by insurance type.

\section{RESULTS}

Table 1 reports summary statistics on variables used in this study, by insurance category at diagnosis. Overall, $28 \%$ of study subjects were in HMOs. The unadjusted distribution for each variable presented (ethnicity, education, neighborhood class, treatment hospital, and stage at diagnosis) differed significantly among patients by type of insurance. The percentage of African Americans and the percentage of least educated among Medicaid and Medicare alone enrollees differs significantly from private FFS enrollees. This dissimilarity strongly suggests the inadvisability of combining these categories (Medicaid, Medicare alone, and private FFS) in comparing outcomes between HMO and FFS enrollees.
The majority of patients in each insurance type were treated at either large or medium/moderately small community hospitals, except for group model HMO patients, who were treated exclusively at HMO-owned hospitals (Table 1). This points up a major distinction between group model and non-group model HMO study subjects.

\section{Stage}

Table 2 presents results from the logistic regression model predicting stage at diagnosis as a function of insurance type and other control variables. Adjusted odds ratios are shown for two outcome categories (Stage I, Stage II $\leq 2.5 \mathrm{~cm}$ ) relative to the baseline category (Stage II $>2.5 \mathrm{~cm}$ ).

Patients in group model HMOs were more likely than those with private FFS insurance to have their tumors diagnosed at Stage I (OR, 1.42; $P=.19$ ), though this difference was not statistically significant at conventional levels. Group model HMO enrollees had greater likelihood of Stage I diagnosis than members of non-group HMOs (OR [group/non-group], 1.64; $P=.13$ ).

\section{Table 2. Likelihood of Early Stage Diagnosis Among 1,080 Female Breast Cancer Cases Age 65 or Older at Diagnosis, by Patient Characteristics, from Multinomial Logistic Regression Model}

\begin{tabular}{|c|c|c|c|}
\hline \multirow[b]{2}{*}{ Characteristic } & \multirow[b]{2}{*}{$n^{\dagger}$} & \multicolumn{2}{|c|}{$\begin{array}{l}\text { Adjusted Odds Ratio }(95 \% \mathrm{Cl}) \text { for Being } \\
\text { Diagnosed at a Specific Stage of Disease* }\end{array}$} \\
\hline & & Stage I $(n=563)$ & $\begin{array}{l}\text { Stage II/ } 2.5 \mathrm{~cm} \text { or } \\
\text { Less }(n=213)\end{array}$ \\
\hline \multicolumn{4}{|l|}{ Health insurance } \\
\hline Medicare/private FFS & 389 & $1.00^{\ddagger}$ & $1.00^{\ddagger}$ \\
\hline Medicare/group model HMO & 186 & $1.42(0.84$ to 2.40$)$ & $1.16(0.62$ to 2.17$)$ \\
\hline Medicare/Medicaid & 165 & $0.50^{\S}(0.31$ to 0.82$)$ & $0.71(0.40$ to 1.25$)$ \\
\hline Other" & 54 & $1.35(0.55$ to 3.26$)$ & $1.09(0.40$ to 3.16$)$ \\
\hline \multicolumn{4}{|l|}{ Ethnicity } \\
\hline White & 773 & $1.00^{\ddagger}$ & $1.00^{\ddagger}$ \\
\hline African American & 106 & 0.50 (0.29 to 0.87$)$ & $0.53(0.27$ to 1.05$)$ \\
\hline Other ${ }^{\sharp}$ & 201 & $1.00(0.64$ to 1.56$)$ & $1.28(0.77$ to 2.14$)$ \\
\hline \multicolumn{4}{|l|}{ Neighborhood class } \\
\hline Non-blue collar & 506 & $1.00^{\dagger}$ & $1.00^{\dagger}$ \\
\hline Blue collar & 574 & $0.70(0.44$ to 1.12$)$ & $0.72(0.42$ to 1.23$)$ \\
\hline \multicolumn{4}{|l|}{ Age at diagnosis } \\
\hline $65-74$ years & 646 & $1.00^{\ddagger}$ & $1.00^{\ddagger}$ \\
\hline 75 years or greater & 434 & $0.72(0.51$ to 1.01$)$ & 0.75 (0.51 to 1.12$)$ \\
\hline
\end{tabular}

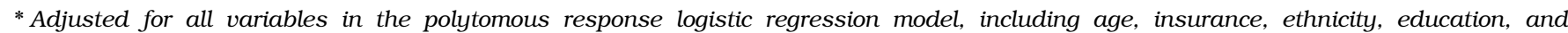
neighborhood class. The baseline outcome category is Stage II patients with tumors $>2.5 \mathrm{~cm}$ in diameter (n = 203). The adjusted odds ratios corresponding to the remaining outcome category (Stage III or IV tumors [n = 101]) were not statistically significant; for the sake of brevity odds ratios for this outcome category are not reported.

${ }^{\dagger}$ Includes patients of all stages. Excluded were 66 patients for whom education and neighborhood class were unknown.

${ }^{\ddagger}$ Reference category.

${ }^{\S} \mathrm{P}<.01$, from logistic regression model.

"Includes 22 patients with private FFS insurance, 15 with private HMO insurance, 5 with Medicaid only, 2 uninsured, and 10 with unknown insurance.

${ }^{\top} \mathrm{P}<.05$, from logistic regression model.

\# The "other" category includes those of Hispanic or Asian ethnicity. 
Table 3. Comparison of Current Study Results with Outcomes Using Potosky et al. Insurance Groupings

\begin{tabular}{|c|c|c|c|}
\hline Outcome Variable and Comparison & Odds Ratio* (95\% Cl) & Risk Ratio* (95\% Cl) & $P$ Value \\
\hline Group model HMO/private $\mathrm{FFS}^{\ddagger}$ & $1.42(0.84$ to 2.40$)$ & & 0.190 \\
\hline \multicolumn{4}{|l|}{ All cause mortality\| } \\
\hline Group model HMO/private $\mathrm{FFS}^{\ddagger}$ & & $0.82(0.53$ to 1.27$)$ & 0.380 \\
\hline Group model $\mathrm{HMO} / \mathrm{FFS}^{\S}$ & & $0.65(0.44$ to 0.98$)$ & 0.037 \\
\hline
\end{tabular}

* Odds ratio refers to the Stage I diagnosis results; risk ratio refers to the survival results.

${ }^{\dagger}$ The analyses of Stage I diagnosis were adjusted for age, insurance type, ethnicity, education, and neighborhood class.

${ }^{\ddagger}$ Current study insurance categories. Results for Stage I diagnosis from Table 2. Survival results modified from Table 5 to exclude treatment and non-group model HMO patients (as per Potosky et al. ${ }^{22}$ ).

${ }^{\S}$ As in the study by Potosky et al., "FFS" insurance includes patients with Medicaid, Medicare alone, and private FFS coverage. Non-group model HMO patients were excluded from these analyses. The survival analysis excluded treatment (as per Potosky et al.).

"The survival analyses for Stage I and II patients were adjusted for age, insurance type, hospital type, education, neighborhood class, ethnicity, and stage.

Of all insurance types, Medicaid patients were least likely to have Stage I tumors (OR, 0.50; $P=.005)$. This is consistent with earlier study findings that Medicaid patients are less likely than those with private insurance to have recent mammograms. ${ }^{53-55}$ Because of the nonexperimental nature of the current dataset and the limited number of patient-level control variables, results attributed to the insurance variable may be partially due to differences in unmeasured patient characteristics correlated with insurance coverage. Indeed, odds ratios associated with the insurance variable should not be interpreted as causal effects.

Whatever the explanation, the large difference in Stage I diagnosis between Medicare patients with private FFS insurance and those with Medicaid argue against measuring HMO performance according to comparisons with a catch-all FFS category. To test for the bias associated with this empirical approach, the stage model was re-estimated with all three types of FFS insurance combined into a single category. Key results from this model, which is similar to that of Potosky et al., ${ }^{22}$ are reported in the top panel of Table 3. Not surprisingly, this specification implies larger and more significant HMO-FFS differences than the specification preferred in the current study.

\section{Treatment}

Figure 1 describes unadjusted data for treatment selected for Stage I /II patients, indicating variation by both type of hospital and time period. Patients at HMOowned hospitals were most likely to be treated with BCS+ (32\% in the two time periods combined). Overall, $8 \%$ of Stage I /II patients were treated with BCS alone (no radiation).

Table 4 presents results from the logistic regression model for treatment selection among Stage I/II patients, showing odds ratios for treatment with BCS+ (vs mastectomy), by hospital type and time period, and by insurance type. Contrary to unadjusted descriptive statistics in
Figure 1, results in Table 4 indicate that in the 19871990 time period only medium/moderately small community hospitals were significantly more likely to treat with $\mathrm{BCS}+$ than large community hospitals (reference group) (OR, 2.60; $P=.006$ ), after adjustment for differences due to stage, insurance, age, ethnicity, and SES variables. By 1991-1993 the significant three-fold increase in likelihood of BCS+ at large community hospitals is most noteworthy (OR, 3.47; $P<.001$ ), but increased use of BCS+ is evident at all types of hospitals.

No model results are presented for Stage I/II patients receiving $\mathrm{BCS}$ without radiation, due to the small number of patients receiving this treatment $(n=86)$. However, age was observed to be a factor related to choice of BCS without
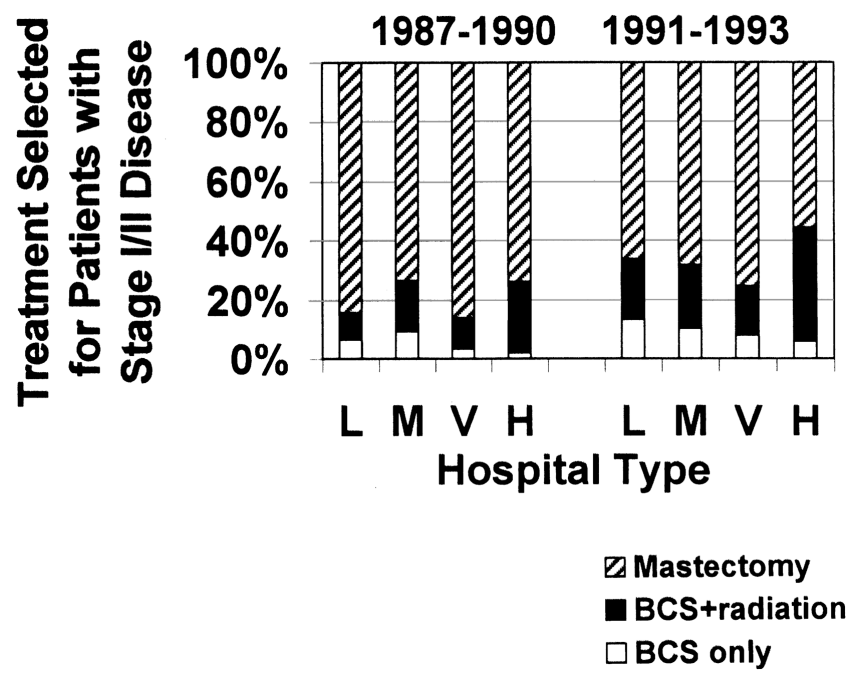

FIGURE 1. Treatment selected for Stage I and II breast cancer patients, by time period at diagnosis and type of hospital where patient received first course of treatment, not adjusted for other factors. L indicates large community hospital; $M$, medium/moderately small community hospital; $V$, very small community/rural hospital; and $\mathrm{H}, \mathrm{HMO}-$ owned hospital. Owing to small numbers, the 33 patients treated at teaching hospitals are not included in the figure. 
Table 4. Likelihood of Receiving Breast-Conserving Surgery and Radiation Among Female Patients with Stage I or II Breast Cancer and Age 65 Years or Older at Diagnosis, by Patient and Hospital Characteristics, from Bivariate Logistic Regression Model

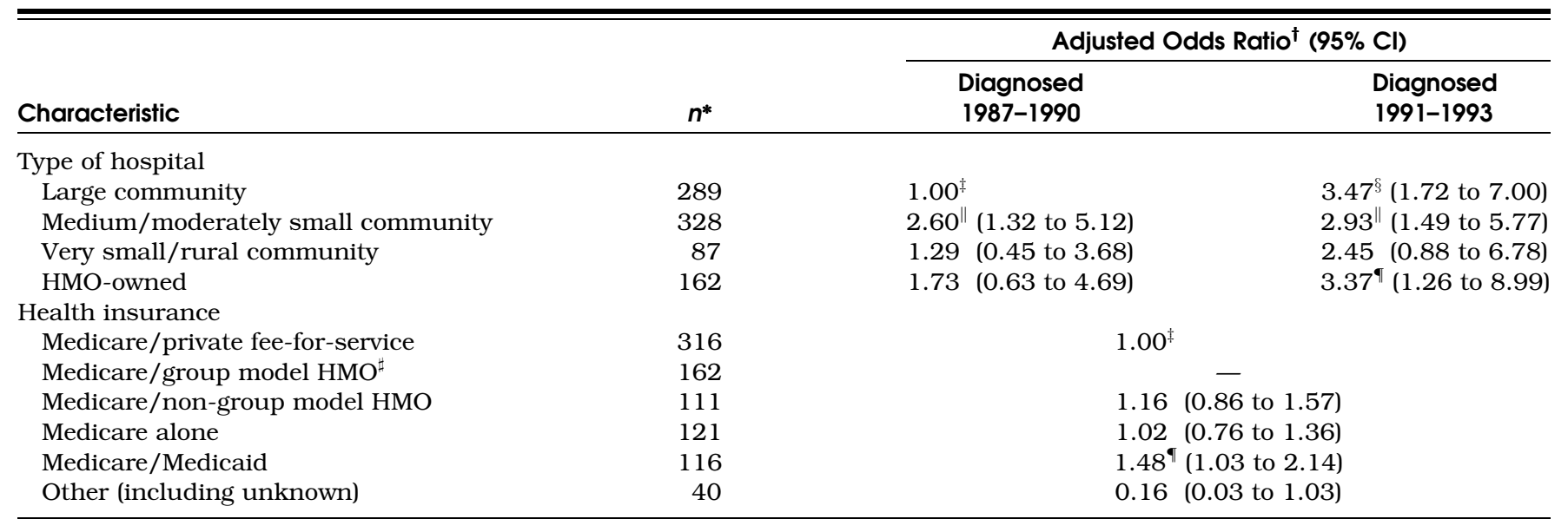

* The purpose of this analysis was to examine factors related to the choice of BCS+ vs mastectomy among Stage I and II patients (for whom $B C S+$ is the NIH recommended treatment) with known education and neighborhood class. For this reason results in this table exclude all Stage III and IV patients, 81 early stage patients receiving BCS without radiation, 2 patients receiving nonsurgical treatment, 2 receiving no treatment, and 3 receiving BCS whose radiation status was unknown. Also excluded were the small number of Stage I and II patients treated at teaching hospitals ( $\mathrm{n}=25)$.

${ }^{\dagger}$ Adjusted for all independent variables included in the dichotomous response logistic regression model, including hospital type, time period, health insurance type, stage at diagnosis, age at diagnosis, ethnicity, education, neighborhood class, and hospital type by time period interaction (the only significant interaction in the model).

${ }^{\ddagger}$ Reference category. There is a single reference category for all hospital-type by time-period odds ratios, namely patients diagnosed at large community hospitals during 1987-1990. This allows for comparisons between various hospital types in 1987-1990 as well as comparisons in 1991-1993 and relative to 1987-1990.

${ }^{\S} \mathrm{P}<.001$, from regression model.

${ }^{\|} \mathrm{P}<.01$, from regression model.

" $\mathrm{P}<.05$, from regression model.

\# Since patients at HMO-owned hospital have only Medicare/group model HMO insurance, hospital type (HMO-owned) was entered into the model for this group of patients and health insurance was not.

radiation; patients aged 80 or more at diagnosis were nearly eight times as likely as those less than age 80 to receive $\mathrm{BCS}$ without radiation as opposed to $\mathrm{BCS}+$ or mastectomy $\left(\chi^{2}, 130.7 ; d f, 2 ; P<.001\right)$.

Generally, the relationship between insurance type and treatment selected was not significant after adjustment for differences in hospital type and other variables considered. The one exception - women with Medicaid - were more likely than those with private FFS coverage to receive $\mathrm{BCS}+(\mathrm{OR}, 1.48 ; P=.04)$.

\section{Survival}

During the ten-year follow-up period there were 393 deaths among study subjects, 171 of these due to breast cancer. Among Stage I and II patients, 104 of the 291 deaths were due to breast cancer. Figure 2, presenting unadjusted survival for Stage I and II patients by type of insurance, suggests that there are significant differences in all-cause mortality among Medicare patients enrolled in the various health plans $(P<.001)$.

Table 5 gives results from the multivariate Cox proportional hazards regression model for both all cause and breast cancer mortality, by type of insurance, stage at diagnosis, treatment, and ethnicity. Each mortality risk ratio (RR) was also adjusted for differences in age, hospital type, and SES variables. An RR greater than 1.00 implies increased risk of dying, relative to the reference category. As expected, both all-cause and breast cancer mortality were significantly related to stage at diagnosis and treatment.

Mortality risks did not differ significantly among patients with private FFS, group model HMO, and nongroup model HMO insurance. However, patients with Medicare alone or with Medicaid had significantly higher all-cause mortality (RR, 1.54, $P=.016$; RR, 1.48, $P=.02$, respectively), and those with Medicare alone had significantly higher breast cancer mortality (RR, 2.36; $P=.007)$ than the private FFS-insured.

In view of the worse all-cause survival of patients with Medicaid compared to private FFS-insured, current study data were used to re-estimate all-cause survival to determine the bias introduced by combining Medicare patients with the three types of FFS insurance (Medicaid, Medicare alone, and private FFS) into a single category. Results from this model, similar to that of Potosky et al. ${ }^{22}$ and given in the bottom panel of Table 3, imply greater and more significant HMO all-cause survival advantage than the preferred result in the current study. 


\section{DISCUSSION}

Earlier research suggested that elderly breast cancer patients in HMOs have a significant advantage over those in FFS Medicare in terms of detection, treatment and survival. The findings of this study suggest an important qualification to this conclusion: among Medicare patients with any type of private insurance, there are no significant differences between those in HMOs and those with FFS supplemental insurance. However, the study reports pronounced differences between Medicare patients with and without private insurance, and this contrast appears to have driven the HMO-FFS differences identified by previous researchers.

This finding has important policy implications. During the current study period, vigorous competition among HMOs for Medicare beneficiaries meant that in many areas, including the present study locale, beneficiaries could obtain HMO coverage at minimal out-of-pocket cost. As a result HMOs were very attractive to Medicare beneficiaries of moderate means who found private supplemental insurance [FFS] prohibitively expensive. More recently,

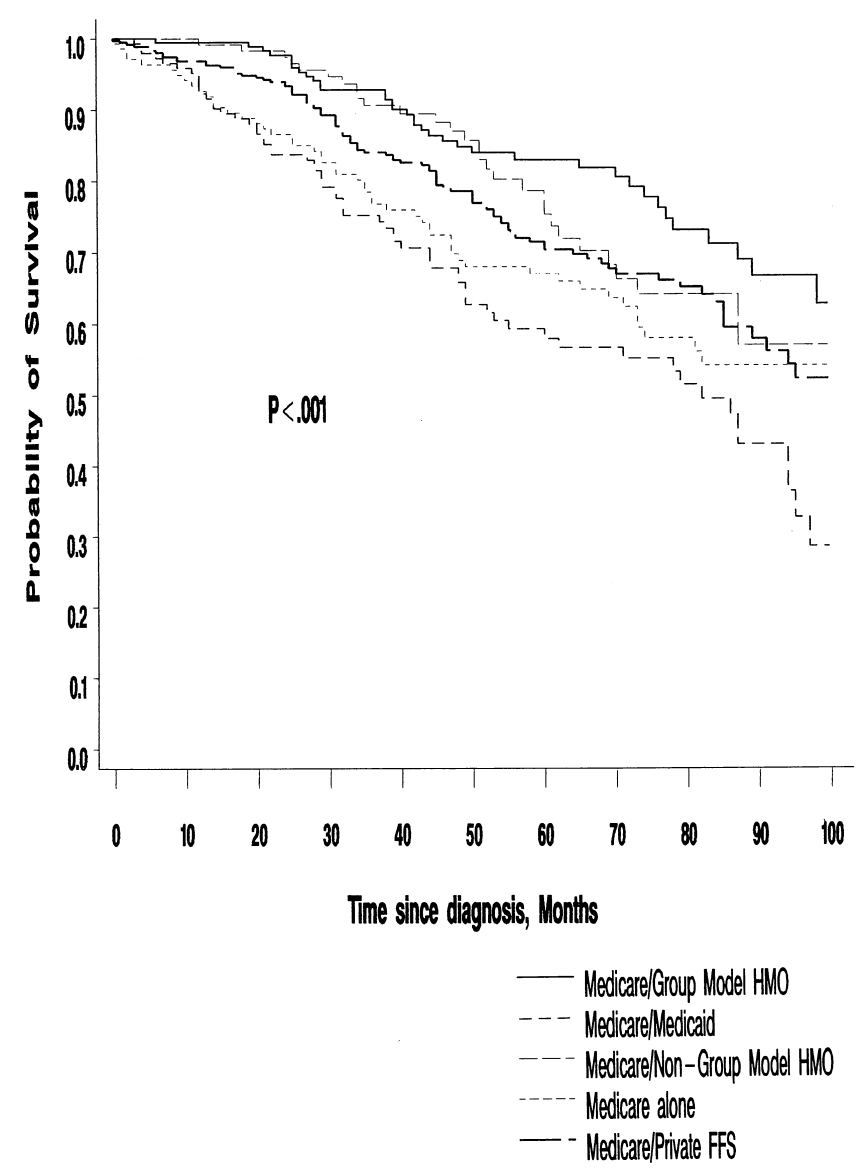

FIGURE 2. Survival of Stage I and II breast cancer patients, by months since diagnosis and health insurance type. The $P$ value refers to the log-rank statistic comparing overall survival among those with various types of health insurance. The small number of patients $(n=46)$ in the "other insurance" category are not included in the figure. reductions in federal payments to HMOs have caused a number of plans to exit the Medicare program, and others to increase premiums to beneficiaries. The similarity in breast cancer outcomes between Medicare HMOs and Medicare/private FFS insurance suggests that patients able to move from the former to the latter will not suffer in terms of the cancer outcomes studied here. However, since HMO coverage does appear to improve outcomes relative to Medicare alone, the contraction of managed care options may have more negative consequences for patients unable to afford, or too sick to qualify for, private FFS supplemental insurance.

Results of this paper regarding the relationship between hospital type and breast cancer outcomes are consistent with a similar study among women under age 65 in northern California. ${ }^{56}$ But they differ from an earlier study ${ }^{17}$ among southern California women of all ages with localized or regional breast cancer, particularly the finding that patients at HMO-owned hospitals were less likely to receive $\mathrm{BCS}+$ than patients at other types of hospitals. These results highlight the fact that delivery system differences can vary considerably across regions.

While this study adds to knowledge of the relationships among insurance coverage, delivery system type, and cancer outcomes, a number of limitations should be acknowledged. As with all studies of this type, the present analysis is based on observational (i.e., non-experimental) data, making it impossible to fully account for differences in patient characteristics that affect both selection into the different insurance categories and the outcome variables. This complicates the interpretation of current results.

Another similarity between this study and others in the literature is the limited geographic scope. An important advantage of focusing on one region is that it allows for collection of additional insurance data. Also, present study comparisons of HMO and FFS patients are not confounded by regional differences in practice patterns. However, an obvious limitation is that these results reflect the behavior of a relatively small number of health plans and hospitals. To the extent that these organizations are unique, current study results may not generalize widely. The fact that the focus is on a region that is both large in size and important with respect to the evolution of its health care market mitigates, but does not eliminate, this shortcoming. Similar research in other geographic regions would be very useful for better understanding of the relationship between financing and delivery system characteristics and treatment outcomes.

No reliable information was available for this study regarding the use of systemic adjuvant therapy. The likelihood that this will bias survival results for Stage I and II patients is believed to be minimal. Comorbidity data were also absent, since the dataset available for this study contained no personal identifiers for individual patients and could not be linked to either hospital discharge files or Medicare claims files which are common sources of comorbidity information. For this reason survival results 
Table 5. Results of Multivariate Survival Analysis (Based on Mortality from all Causes and Breast Cancer Mortality Only) by Prognostic Factors, Among Stage I or Stage II Breast Cancer Patients Aged 65 or Older and Diagnosed from 1987 Through 1993

\begin{tabular}{|c|c|c|c|}
\hline & & All Cause Mortality & Breast Cancer Mortality \\
\hline Prognostic Factor & $n$ & Risk Ratio* (95\% Cl) & Risk Ratio* (95\% Cl) \\
\hline \multicolumn{4}{|l|}{ Health insurance at diagnosis } \\
\hline Medicare/private FFS & 356 & $1.00^{\dagger}$ & $1.00^{\dagger}$ \\
\hline Medicare/group model HMO & 170 & $0.86(0.56$ to 1.31$)$ & $1.91(0.98$ to 3.73$)$ \\
\hline Medicare/non-group model HMO & 119 & $0.86(0.56$ to 1.31$)$ & $1.64(0.83$ to 3.26$)$ \\
\hline Medicare alone & 140 & $1.54^{\ddagger}(1.08$ to 2.18$)$ & $2.36^{\S}(1.27$ to 4.41$)$ \\
\hline Medicare/Medicaid & 148 & $1.48^{\ddagger}(1.06$ to 2.07$)$ & $1.67(0.88$ to 3.17$)$ \\
\hline Other (including unknown) & 46 & $0.98(0.51$ to 1.91$)$ & $1.21(0.41$ to 3.59$)$ \\
\hline \multicolumn{4}{|l|}{ Stage at diagnosis } \\
\hline I & 563 & $1.00^{\dagger}$ & $1.00^{\dagger}$ \\
\hline II $\leq 2.5 \mathrm{~cm}$ in diameter & 213 & $1.93^{\|}(1.43$ to 2.61$)$ & $4.19^{\|}(2.46$ to 7.13$)$ \\
\hline II $>2.5 \mathrm{~cm}$ in diameter & 203 & $2.66^{\|}(1.99$ to 3.56$)$ & $5.75^{\|}(3.39$ to 9.76$)$ \\
\hline \multicolumn{4}{|l|}{ Treatment } \\
\hline Breast-conserving surgery & 83 & $2.84^{\|}(1.70$ to 4.75$)$ & $0.62(0.13$ to 2.94$)$ \\
\hline Breast-conserving surgery + radiation & 188 & $1.00^{\dagger}$ & $1.00^{\dagger}$ \\
\hline Mastectomy & 701 & $1.48(0.99$ to 2.20$)$ & $1.55(0.79$ to 3.05$)$ \\
\hline Other & 7 & - & - \\
\hline \multicolumn{4}{|l|}{ Ethnicity } \\
\hline White & 709 & $1.00^{\dagger}$ & $1.00^{\dagger}$ \\
\hline African American & 86 & $1.38(0.95$ to 2.01$)$ & $1.64(0.92$ to 2.91$)$ \\
\hline Other ${ }^{\sharp}$ & 184 & $0.64^{\S}(0.45$ to 0.90$)$ & $0.80(0.47$ to 1.35$)$ \\
\hline
\end{tabular}

* From the Cox regression analysis with the following independent variables: health insurance type, stage at diagnosis, treatment, ethnicity, hospital type, age, education, and neighborhood class.

${ }^{\dagger}$ Reference category. RR $>1$ indicates increased risk of dying, compared with the reference category.

${ }^{\ddagger} \mathrm{P}<.05$.

${ }^{\S} \mathrm{P}<.01$

${ }^{\|} \mathrm{P}<.001$.

"Includes 2 patients receiving nonsurgical treatment, 2 receiving no treatment, and 3 receiving BCS whose radiation treatment status was unknown. Owing to the small number of patients in this category, no risk ratios or confidence intervals are shown.

${ }^{\sharp}$ Includes patients of Hispanic or Asian ethnicity.

in this study should be interpreted with caution. However, comorbidity adjustments are believed to have minimal effect on mortality risk ratios associated with insurance after adjustments have been made for age, ethnicity, education, neighborhood class, hospital type, stage, and treatment - as a previous study of Medicare patients with breast cancer suggests. ${ }^{22}$ Secondly, women aged 80 or more with Stage I breast cancer and two or more comorbid conditions reportedly are likely to receive BCS, and their probability of receiving radiation therapy tends to decline rapidly with age. $^{21}$ The significant all-cause mortality associated with BCS alone in Table 5 may in part be a result of the presence of comorbid conditions among the 83 women receiving $\mathrm{BCS}$ alone, approximately two thirds of whom were aged 80 or more. In the future better data will allow these limitations to be addressed.

Despite its limitations this study clarifies the relationship of insurance type and hospital type to breast cancer outcomes among Medicare patients. The need for greater use of BCS+ among early stage Medicare patients suggests that physician education on the NIH-recommended treatment methods continued to require attention of the medical profession after the early 1990s. While rates of BCS+ use in the current study region are expected to have increased in the mid- to late-1990s, large variation by hospital type is likely to persist.
Cancer incidence data have been provided by the California Department of Health Services and its agent, the Public Health Institute, as part of its statewide cancer reporting program, mandated by Health and Safety Code Section 103875 and 103885. The ideas and opinions expressed herein are those of the authors, and no endorsement of the State of California, Department of Health Services or the Public Health Institute, is intended or should be inferred.

The authors also wish to acknowledge the assistance of the Cancer Surveillance Program, Region 3, particularly Vincent Caggiano, MD, Sutter Cancer Center Medical Director and Principal Investigator; Kathleen Davidson-Allen, former Director; and Cheryl Tatum, Study Coordinator.

This research was supported by grant number CA-71236 from the National Cancer Institute. Its contents are solely the responsibility of the authors and do not necessarily represent the official views of the National Cancer Institute.

Dr. Katterhagen was formerly Medical Director, Cancer Program and Breast Center, Mills-Peninsula Hospital, Burlingame, Calif.

\section{REFERENCES}

1. Hoechst Marion Roussel. HMO-PPO/Medicare-Medicaid Digest. Managed Care Digest Series 1997. Kansas City, Mo: Hoechst Marion Roussel; 1997. 
2. Hoechst Marion Roussel. HMO-PPO/Medicare-Medicaid Digest. Managed Care Digest Series 1998. Kansas City, Mo: Hoechst Marion Roussel; 1998.

3. Ries LAG, Kosary CL, Hankey BF, Miller BA, Edwards BK, eds. SEER Cancer Statistics Review, 1973-1995. Bethesda, Md: National Cancer Institute; 1998.

4. Ries LAG, Kosary CL, Hankey BF, Harras A, Miller BA, Edwards BK, eds. SEER Cancer Statistics Review, 1973-1993: Tables and Graphs. Bethesda, Md: National Cancer Institute; 1996.

5. Morris CR, Cohen R, Perkins CI, Allen M, Kwong SL, Schlag R, Wright WE. Cancer in California: 1988-1996. Sacramento, Calif: California Department of Health Services, Cancer Surveillance Section; March 1999

6. Breen N, Kessler L. Changes in the use of screening mammography: evidence from the 1987 and 1990 National Health Interview Surveys. Am J Public Health. 1994;84:62-7.

7. Morris CR, Wright WE, eds. Breast Cancer in California. Sacramento, Calif: California Department of Health Services, Cancer Surveillance Section; March 1996.

8. Centers for Disease Control. Self-reported use of mammography among women aged $\geq 40$ years — United States, 1989 and 1995. MMWR. 1997;46:937-41.

9. Veronesi U, Saccozzi R, DelVecchio M, et al. Comparing radical mastectomy with quadrantectomy, axillary dissection, and radiotherapy in patients with small cancer of the breast. N Engl J Med. 1981;305:6-11.

10. Sarrazin D, Le M, Rouesse J, et al. Conservative treatment versus mastectomy in breast cancer tumors with macroscopic diameter of 20 millimeters or less. Cancer. 1984;53:1209-13.

11. Fisher B, Bauer M, Margolese R, et al. Five-year results of a randomized clinical trial comparing total mastectomy and segmental mastectomy with or without radiation. N Engl J Med. 1985;312:665-73.

12. NIH Consensus Conference. Treatment of early-stage breast cancer. JAMA. 1991;265:391-5.

13. Lazovich D, White E, Thomas DB, Moe RE. Underutilization of breast-conserving surgery and radiation therapy among women with stage I or II breast cancer. JAMA. 1991;266:3433-8.

14. Farrow DC, Hunt WC, Samet JM. Geographic variation in the treatment of localized breast cancer. N Engl J Med. 1992;326: 1097-101.

15. Hunter CP, Redmond CK, Chen VW, et al. Breast cancer: factors associated with stage at diagnosis in black and white women. J Natl Cancer Inst. 1993;85:1129-37.

16. Eley JW, Hill HA, Chen VW, Austin DF, et al. Racial differences in survival from breast cancer. Results of the National Cancer Institute Black/White Cancer Survival Study. JAMA. 1994;272: 947-54.

17. Lee-Feldstein A, Anton-Culver H, Feldstein PJ. Treatment differences and other prognostic factors related to breast cancer survival. Delivery systems and medical outcomes. JAMA. 1994;271:1163-8.

18. Riley GF, Potosky AL, Lubitz JD, Brown ML. Stage of cancer at diagnosis for Medicare HMO and fee-for-service enrollees. Am J Public Health. 1994;84:1598-604.

19. Foster RS, Farwell ME, Costanza MC. Breast-conserving surgery for breast cancer: patterns of care in a geographic region and estimation of potential applicability. Ann Surg Oncol. 1995;2: 275-80.

20. Johantgen ME, Coffey RM, Harris DR, Levy H, Clinton JJ. Treating early-stage breast cancer: hospital characteristics associated with breast-conserving surgery. Am J Public Health. 1995; 85: $1432-4$.

21. Ballard-Barbash R, Potosky AL, Harlan LC, Nayfield SG, Kessler LG. Factors associated with surgical and radiation therapy for early stage breast cancer in older women. J Natl Cancer Inst. 1996;88:716-26.
22. Potosky AL, Merrill RM, Riley GF, Taplin SH, Barlow W, Fireman VH, Ballard-Barbash R. Breast cancer survival and treatment in health maintenance organization and fee-for-service settings. J Natl Cancer Inst. 1997;89:1683-91.

23. Riley GF, Potosky AL, Klabunde CN, Warren JL, Ballard-Barbash $\mathrm{R}$. Stage at diagnosis and treatment patterns among older women with breast cancer: an HMO and fee-for-service comparison. JAMA. 1999;281:720-4.

24. Osteen RT, Winchester DP, Hussey DH, et al. Insurance coverage of patients with breast cancer in the 1991 Commission on Cancer Patient Care evaluation study. Ann Surg Oncol. 1994; 1:462-7.

25. Gabel R. Ten ways HMOs have changed during the 1990s. Health Aff. 1997;16:134-45.

26. Nattinger AB, Gottlieb MS, Veum J, Yahnke D, Goodwin JS. Geographic variation in the use of breast-conserving treatment for breast cancer. N Eng J Med. 1992;326:1102-7.

27. Nattinger AB, Gottlieb MS, Hoffman RG, Walker AP, Goodwin JS. Minimal increase in use of breast-conserving surgery from 1986 to 1990. Med Care. 1996;34:479-89.

28. Young WW, Marks SM, Kohler SA, Hsu AY. Dissemination of clinical results. Mastectomy versus lumpectomy and radiation therapy. Med Care. 1996;34:1003-17.

29. Kotwall CA, Covington DL, Rutledge R, Churchill MP, Meyer AA. Patient, hospital, and surgeon factors associated with breast conservation surgery. A statewide analysis in North Carolina. Ann Surg. 1996;224:419-29.

30. Perkins CI, Morris CR, Wright WE. Cancer Incidence and Mortality in California by Race/Ethnicity, 1988-1993. Sacramento, Calif: California Department of Health Services, Cancer Surveillance Section; March 1996.

31. Percy C, Van Holten V, Muir C, eds. International Classification of Diseases for Oncology. 2nd ed. Geneva, Switzerland: World Health Organization; 1990.

32. American Joint Committee on Cancer. Manual for Staging of Cancer. 4th ed. Philadelphia, Pa: JB Lippincott, 1992.

33. Seiffert J, ed. SEER Program Comparative Staging Guide for Cancer, Version 1.1. Bethesda, Md: National Institutes of Health; 1993. NIH publication 93-3640.

34. Hayward RA, Shapiro MF, Freeman HE, Corey CR. Who gets screened for cervical and breast cancer? Results from a new national survey. Arch Intern Med. 1988;148:1177-81.

35. Krieger N. Social class and the black/white crossover in the agespecific incidence of breast cancer: a study linking census-derived data to population-based registry records. Am J Epidemiol. 1990;131:804-14.

36. Lerman C, Rimer B, Trock B, Balshem A, Engstrom PF. Factors associated with repeat adherence to breast cancer screening. Prev Med. 1990;19:279-90.

37. Mandelblatt $J$, Andrews H, Kerner J, Zauber A, Burnett W. Determinants of late stage diagnosis of breast and cervical cancer: the impact of age, race, social class, and hospital type. Am J Public Health. 1991;81:646-9.

38. Wells BL, Horm JW. Stage at diagnosis in breast cancer: race and socioeconomic factors. Am J Public Health. 1992;82:1383-5.

39. Katz SJ, Hofer TP. Socioeconomic disparities in preventive care persist despite universal coverage. Breast and cervical cancer screening in Ontario and the United States. JAMA. 1994;272: 530-4.

40. Breen N, Figueroa JB. Stage of breast and cervical cancer diagnosis in disadvantaged neighborhoods: a prevention policy perspective. Am J Prev Med. 1996;12:319-36.

41. Michalski TA, Nattinger AB. The influence of black race and socioeconomic status on the use of breast-conserving surgery for Medicare beneficiaries. Cancer. 1997;79:314-9.

42. Krieger N, Williams DR, Moss NE. Measuring social class in US public health research: concepts, methodologies, and guidelines. Annu Rev Public Health. 1997;18:341-78. 
43. State of California, Department of Health Services, Medi-Cal Policy Division. Hospital Peer Grouping. Sacramento, Calif: State of California, Department of Health Services; 1991.

44. American Hospital Association. 1990 American Hospital Association Guide to the Health Care Field. Chicago, Ill: American Hospital Association; 1991.

45. SAS Institute Inc. SAS/STAT User's Guide.Version 6. Cary, NC: SAS Institute; 1990.

46. SAS Institute Inc. SAS/STAT Software: Changes and Enhancements through Release 6.11. Cary, NC: SAS Institute; 1996.

47. Hosmer DW, Lemeshow S. Applied Logistic Regression. New York, NY: Wiley; 1989.

48. Stokes ME, Davis CS, Koch GG. Categorical Data Analysis Using the SAS System. Cary, NC: SAS Institute; 1995.

49. Cox DR. Regression models and life tables. J R Stat Soc B. 1972;34:187-220.

50. Allison PD. Survival Analysis Using the SAS System: A Practical Guide. Cary, NC: SAS Institute; 1995.
51. Kaplan EL, Meier PL. Nonparametric estimation from imcomplete observations. J Am Stat Assoc. 1958;53:457-81.

52. Mantel N, Haenszel W. Statistical aspects of the analysis of data from retrospective studies of disease. J Natl Cancer Inst. 1959;22:719-48.

53. Makuc DM, Freid VVM, Parsons PE. Health insurance and cancer screening among women. Advance Data from Vital and Health Statistics; no. 254. Hyattsville, Md: National Center for Health Statistics; 1994.

54. Blustein J. Medicare coverage, supplemental insurance, and the use of mammography by older women. N Engl J Med. 1995;332: 1138-42.

55. Potosky AL, Breen N, Braubard BI, Pearsons PE. The association between health care coverage and the use of cancer screening tests. Med Care. 1998;36:257-70.

56. Lee-Feldstein A, Feldstein PJ, Buchmueller T, Katterhagen G. The relationship of HMOs, health insurance, and delivery systems to breast cancer outcomes. Med Care. 2000;38:705-18.

\section{JGIM Website - Visit us online today!}

Please visit the JGIM World-Wide Website:

http://www.blackwellscience.com/journals 